\title{
El mapuche hablado en Curarrehue: fonemas SEGMENTALES, FONOTAXIS Y COMPARACIÓN CON OTRAS VARIEDADES*
}

\author{
Chery Pérez ${ }^{* *}$ \\ Gastón Salamanca***
}

\section{Resumen}

Este artículo presenta los fonemas segmentales y alófonos del mapuzungun hablado en Curarrehue. El marco de referencia escogido es el descriptivismo norteamericano. En el aspecto metodológico, la muestra está constituida por 10 colaboradores adultos (entre 30 y 60 años), hablantes de mapuzungun, pertenecientes a la comuna de Curarrehue, a los cuales se les aplicó una lista léxica ad hoc que relevó los fonemas y alófonos de la variante en estudio. En cuanto a los principales resultados, destaca el predominio de las realizaciones áfonas de los fonemas /f / y / $\theta /$, la asignación de estatus de fonema a las interdentales [t]], [n], [l] y a los fonos fricativos [J] y [Y]; y la baja cantidad de interferencias del español.

Palabras clave: Mapuche, fonología del mapuche, dialectología del mapuche, mapuche hablado en Curarrehue.

\section{MAPUCHE SPOKEN IN LONQUIMAY: SEGMENTAL PHONEMES, PHONOTAXIS AND COMPARISON WITH OTHER VARIETIES}

\begin{abstract}
This paper presents the segmental phonemes and allophones of the spoken mapuzungun in Curarrehue. The chosen reference frame is the American descriptivism. In terms of methodology, the sample consists of 10 collaborators, adults (between 30 and 60 years), mapuzungun speakers, belonging to Curarrehue, to whom an ad hoc lexical list was applied, which relieved the phonemes and allophones of the studied variant. The main results were the predominance of voiceless realizations of the phonemes $/ f /$ and $/ \theta /$, the phonemic status of the interdentals [t]], [n], [l] and fricative phones [j] and [V], and the low number of interferences of the Spanish is highlighted.
\end{abstract}

Keywords: Mapuche, Mapuche phonology, Mapuche dialectology, Spoken Mapuche in Currarehue.

* Este artículo contiene los principales resultados de la tesis de Magíster en Lingüística Fonemas Segmentales y alófonos del mapuzugun hablado en Curarrehue y determinación de los grafemas más idóneos para su representación.

** Chilena. Magíster en Lingüística por la Universidad de Concepción, Concepción, Chile. cheryperez@ udec.cl

*** Chileno. Doctor en Lingüística por la Universidad de Concepción. Académico de la Facultad de Humanidades y Arte del Departamento de Español, Universidad de Concepción, Concepción, Chile. gaston.salamanca@gmail.com. 


\section{Introducción}

La lengua hablada por los mapuches se denomina mapuzungu(n) o chezungu(n). Su uso, en Chile, se extiende entre la VIII y $\mathrm{X}$ regiones y, aunque es una de las lenguas más estudiadas en Sudamérica (Lagos, 1981), aún existen localidades donde sus descripciones son precarias o inexistentes. En este contexto, el artículo que presentamos tiene como objetivo fundamental describir, de acuerdo con el método distribucional, las características del nivel fónico segmental del mapuzungun hablado por los pewenches de Curarrehue, dado que, por ejemplo, el estudio dialectológico del mapuzungun desarrollado por Croese (1980) no contiene datos provenientes de esta localidad, aunque en este sector cordillerano es posible encontrar hablantes activos de la lengua.

La metodología empleada en la investigación considera el análisis distribucional de los segmentos (Pike, 1947; Burquest, 2009), el cual fue complementado con apoyo tecnológico (grabaciones de audio y video). La muestra estuvo conformada por diez hablantes activos de mapuzungun adultos, y el instrumento aplicado a dicha muestra fue una lista léxica ad hoc, que relevó los fonemas y alófonos de la variante en estudio.

En este contexto, conviene señalar que el hecho de conocer y describir variantes que no han sido estudiadas constituye la base para el desarrollo de posibles estudios acerca, por ejemplo, de la vitalidad de la lengua, adquisición del mapuzungun, etc.

En términos más específicos, los objetivos de este artículo son presentar los fonemas segmentales, y sus respectivas realizaciones alofónicas, de la variante del mapuzungun hablada por los pewenches de Curarrehue; establecer la pauta fonotáctica de la variante del mapuzungun hablada en dicha zona; y determinar los rasgos afines y diferenciadores con respecto al mapuzungun hablado en dos localidades de Alto Biobío.

\section{Marco de referencia}

El marco de referencia en que se inscribe nuestra investigación es el que sustenta la gran mayoría de las descripciones fonético-fonológicas clásicas del mapudungun ${ }^{1}$ (el descriptivismo norteamericano), 
desarrollado, entre muchos otros, por Bloomfield (1933), Gleason (1961) y, en el ámbito de la fonología, por Pike (1947). Las nociones de fonema y alófono, contexto, etc., las cuales se han instalado de manera estable en la disciplina, son definidas de acuerdo con este modelo.

Este fondo teórico-metodológico surge en el contexto de una «lingüística de salvataje» de lenguas amenazadas, por lo cual su énfasis principal ha estado en los procedimientos del trabajo de campo y el análisis de datos.

Así, en este artículo se siguen los principios teórico-metodológicos propuestos por Pike (1947) y Burquest (2009), los cuales se presentan paso a paso en Echeverría (1964). La noción de fluctuación de fonemas se sigue de acuerdo con Salas (2006[1992]); y la de transferencias, de acuerdo a como ha sido aplicada por Henríquez (2004 y 2013).

\section{Metodología}

\subsection{Zona}

Los datos fueron recogidos en la comuna de Curarrehue. Esta comuna se encuentra ubicada a $40 \mathrm{Km}$ de la frontera con Argentina por el Paso Mamuil Malal. Su población es principalmente de origen mapuche y las actividades económicas principales son la industria maderera y la ganadería.

A continuación, se presenta un mapa con la finalidad de dar a conocer el sector específico donde se recolectaron los datos, el cual ha sido señalado con un círculo.

Figura 1. Mapa sector de Curarrehue ${ }^{2}$

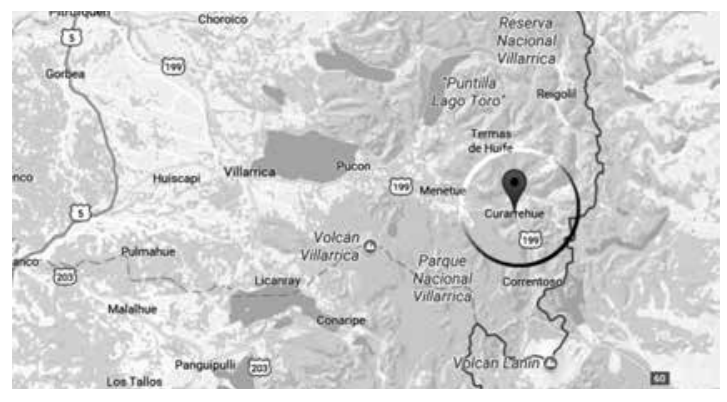

$2 \quad$ Extraído de Google Maps. 


\subsection{Colaboradores}

Para la presente investigación, se llevó a cabo un registro de datos de 10 colaboradores adultos (entre 30 y 60 años), hablantes de mapuzungun, pertenecientes a la comuna de Curarrehue y sus comunidades indígenas aledañas, los cuales fueron contactados por el Educador de Lengua y Cultura Indígena (ELCI) de la escuela Ruka Manke, sector Reigolil, Neftalí Carinao.

La muestra en cuestión se puede etiquetar como intencionada y no aleatoria, pues se busca que los participantes evidencien ciertas características fundamentales como buen dominio de la lengua, ascendencia entre sus pares, etc. En este contexto, fue fundamental contar con el apoyo de don Neftalí al momento de escoger los hablantes.

\subsection{Instrumento}

Las entrevistas se llevaron a cabo en 3 viajes a la zona. En la primera, se entrevistó a 2 colaboradores; en la segunda, a 3; y en la tercera, la más extensa, a los 5 colaboradores restantes. La entrevista fue elaborada sobre la base de una lista léxica que focaliza, entre otros aspectos, el estatus de las consonantes interdentales y la sonoridad de las consonantes fricativas planas. En este contexto se tomó como referencia la lista léxica utilizada en Salamanca (1997).

\subsection{Registro de la información}

Para el registro de audio se utilizó una grabadora TASCAM DR-100. Además, con la finalidad de tener un registro visual de la articulación de los enunciados emitidos por los colaboradores, cada sesión fue filmada con una cámara HANDYCAM SONY DCR-DVD108/DVD308 / DVD608/DVD708; así se pudo determinar las características de los segmentos puestos en foco. Este tipo de análisis fue particularmente útil para determinar las consonantes interdentales. Después del trabajo de campo, se procedió a analizar detenidamente los registros y realizar la transcripción. Esta se llevó a cabo de acuerdo con los símbolos propuestos por el Alfabeto Fonético Internacional (AFI, versión actualizada en 2005). 


\section{Fonemas segmentales del mapuche hablado de Curarrehue}

\subsection{Fonemas vocálicos}

Se han identificado los siguientes: /a, e, i, o, u, ə/.

\subsubsection{Fonema / a /}

Se realiza como [a]: vocoide baja, central, no redondeada, silábica. Por ejemplo, [a[n].'[t]

\subsubsection{Fonema /e/}

Se realiza como [e]: vocoide media, anterior, no redondeada, silábica. Por ejemplo, [e.'pu] 'dos', [pu.'kiem] 'invierno', [ [an.'we] 'chancho'.

\subsubsection{Fonema / i/}

Se realiza como [i]: vocoide alta, anterior, no redondeada, silábica.

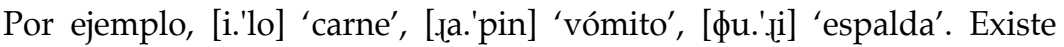
fluctuación de fonemas entre $/ \mathrm{i} / \sim /$ / / y $/ \mathrm{i} / \sim / \mathrm{u} /$, lo cual es posible observar en los siguientes ejemplos: /po.ni/ /po.nə / 'papa' (en este caso, se observa que, por su direccionalidad, la fluctuación puede asociarse con una vitalidad de la sexta vocal); / ku.ni.fal/ / ku.nu.fal/ 'huérfano', respectivamente.

\subsubsection{Fonema /o/}

Se realiza como [o]: vocoide media, posterior, redondeada, silábica. Por ejemplo, ['koф.kie] 'pan', ['ko] 'agua'. Existe fluctuación de fonemas entre $/ \mathrm{o} / \mathrm{y}$ la semiconsonante $/ \mathrm{w} /$, como se observa en el siguiente ejemplo: / kə.Өa.o/ / kə.Өaw/ 'trabajo'.

\subsubsection{Fonema / u/}

Se realiza como $[u]$ : vocoide alta, posterior, redondeada, silábica. Por ejemplo, [u.'фi.ja] ‘oveja', [pa.'jun] ‘bigote', ['wen.tşu] ‘hombre’.

\subsubsection{Fonema / / /}

Posee dos realizaciones alofónicas: 
- [u]: vocoide alta, posterior, no redondeada, silábica, la cual se presenta en posición inicial de palabra y después de consonante velar o retrofleja. Por ejemplo, [u.'Jəm] 'pájaro', [ku.'la] 'tres', [yu.'£u] 'zorro'.

- [ə]: vocoide media, central, no redondeada, silábica. Se presenta en otros contextos. Por ejemplo, ['mə.ta] 'cuerno', [na.'yan,to] 'atardecer'.

Existe fluctuación de fonemas entre $/ \mathrm{\partial} / \sim / \mathrm{u} / \mathrm{y} / \mathrm{\partial} / \sim / \mathrm{i} /$. Por ejemplo: / țo.mə/ / țo.mu/ 'nube' y /ə.jim/ /i.jim/ 'pájaro', respectivamente. Este tipo de fluctuaciones también se pueden interpretar como casos de influencia de la lengua mayoritaria en la lengua vernácula.

Además, se observa la presencia de un desarrollo consonántico delante de [u], como en [үu.'Jəm].

\subsection{Cuadro de fonos y fonemas vocálicos}

\subsubsection{Fonos vocálicos silábicos}

Tabla 1. Fonos vocoides silábicos de Curarrehue

\begin{tabular}{|c|c|c|c|c|}
\hline & Anteriores & Centrales & \multicolumn{2}{|c|}{ Posteriores } \\
\hline & & & No labializadas & Labializadas \\
\hline Altas & {$[\mathrm{i}]$} & & {$[\mathrm{u}]$} & {$[\mathrm{u}]$} \\
\hline Medias & {$[\mathrm{e}]$} & {$[ə]$} & & {$[\mathrm{o}]$} \\
\hline Bajas & & {$[\mathrm{a}]$} & & \\
\hline
\end{tabular}

\subsubsection{Fonemas vocálicos}

Tabla 2. Fonemas vocálicos de Curarrehue

\begin{tabular}{|c|c|c|c|}
\hline & Anteriores & Centrales & Posteriores \\
\hline Altas & $/ \mathrm{i} /$ & & $/ \mathrm{u} /$ \\
\hline Medias & $/ \mathrm{e} /$ & $/ \mathrm{a} /$ & $/ \mathrm{o} /$ \\
\hline Bajas & & $/ \mathrm{a} /$ & \\
\hline
\end{tabular}

\subsection{Fonemas consonánticos}

Se han identificado los siguientes: / $\mathrm{p}, \mathrm{t}, \mathrm{t}, \mathrm{k}, \mathrm{f}, \theta, \mathrm{t}, \mathrm{t} \int, \int, \mathrm{ts}, \mathrm{m}, \mathrm{n}, \mathrm{n}, \mathrm{n}$, $n_{n} 1, l_{n} \Lambda /$. 


\subsubsection{Fonema / p/}

Se realiza como [p]: contoide obstruyente, oclusiva, bilabial, sorda, oral. Por ejemplo, [pa.'ta.ka] 'cien', [t]a.'pa $\theta$ ] 'greda'.

\subsubsection{Fonema / $/$}

Se realiza como [t]: contoide obstruyente, oclusiva, alveolar, sorda, oral. Por ejemplo, [ta.'kun] 'ropa', [nji.Ka.'tun]' rogativa'. Existe fluctuación de fonemas entre $/ \mathrm{t} / \sim / \mathrm{t} /$. Por ejemplo: /to.ki/ / to.ki/ 'hacha'. La direccionalidad de la fluctuación podría asociarse con la vitalidad de [t].

\subsubsection{Fonema / t $/$}

Se realiza como [t]: contoide obstruyente, oclusiva, interdental, sorda, oral. Por ejemplo, ['tol] 'frente', [mə.'ta] 'cuerno'.

Podemos observar que tanto $[\mathrm{t}]$ como $[\mathrm{t}]$ aparecen en posición inicial y entre vocales; por lo tanto, ambas se encuentran en distribución contrastante por ambiente análogo, y, en consecuencia, pertenecen a fonemas diferentes. En el caso de $/ t /$, se identifica una alta frecuencia de uso $(74 \%)$ y una consistente ocurrencia en palabras como 'frente', 'cuerno', piojo', 'hoy', 'sol' y 'atardecer'.

Existe fluctuación de fonemas entre $/ \mathrm{t} / \sim / \mathrm{t} /$. Por ejemplo, / fa.tfan, to/ / / fa.tfan.tə/ 'hoy', /an,to/ / [an, 'to] 'sol'. Esta fluctuación también se puede interpretar como una influencia de la lengua mayoritaria en la lengua vernácula.

\subsubsection{Fonema / k/}

Posee dos realizaciones alofónicas en distribución complementaria:

- [k]: contoide obstruyente, oclusiva, velar, sorda, oral. Ocurre ante

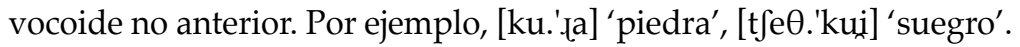

- [ki]: contoide obstruyente, oclusiva, postpalatal, sorda, oral. Ocurre ante vocoide anterior. Por ejemplo, [kin.'tu] 'buscar', [to.'ki] 'hacha'.

\subsubsection{Fonema / ts /}

Se realiza únicamente como [ts]: contoide africada, alveolar retrofleja, sorda, oral. Por ejemplo, ['tşa.pj] 'aji', [fi.'tşun] 'humo'. A diferencia de otras zonas, no presenta alofonía. 
En nuestros datos, también ocurre la secuencia [tr], la cual es atribuible al contacto con el español. Sin embargo, su frecuencia es muy baja (1\%).

\subsubsection{Fonema $/ \mathrm{t} \int /$}

Este fonema se realiza como [tf]: contoide africada, alveopalatal, sorda, oral. Por ejemplo, [tfa.' $\theta \mathrm{i}]$ 'sal', [ka.'tfu] 'pasto'. Hay fluctuación de fonemas entre $/ \mathrm{t} f / \sim / \mathrm{t} / \mathrm{y} / \mathrm{t} \int / \sim / \mathrm{s} /$. Por ejemplo, $/ \mathrm{t}$ a. $\theta \mathrm{i} / \sim / \mathrm{ta} . \mathrm{j} /$ 'sal', / ke.tfu/ / ke.ju/ 'cinco', respectivamente.

\subsubsection{Fonema /f/}

Tiene tres realizaciones alofónicas en variación libre: [f]: contoide fricativa plana, labiodental, sorda, oral; $[\phi]$ : contoide fricativa, bilabial, sorda, oral; [v]: contoide fricativa plana, labiodental, sonora, oral. Por

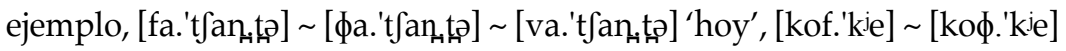

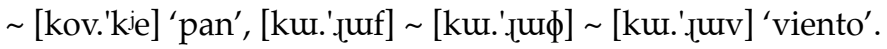

Como se observa en los ejemplos, los tres alófonos alternan en variación libre, aunque dentro de la muestra predominan significativamente las realizaciones sordas [f] $(63 \%)$ y $[\phi](35 \%)$; por esta razón, se ha seleccionado la realización labiodental sorda [f] como la forma básica del fonema.

\subsubsection{Fonema / $\theta /$}

Este fonema tiene tres realizaciones alofónicas en variación libre: $[\theta]$ : contoide fricativa plana, interdental, sorda, oral; [ð]: contoide fricativa plana, interdental, sonora, oral; [s]: contoide fricativa, ápicoalveolar, sorda, oral. Por ejemplo, [Өo.'mo] [ðo.'mo] 'mujer', [ma.'wi.Өa] [ma.'wi.ða] [ma.'wi.sa] 'montaña' y ['tfo $\theta] \sim$ ['tfoð] ['tfos] 'amarillo'.

En cuanto a la frecuencia de aparición, al igual que en el caso del fonema $/ \mathrm{f} /$, el alófono sordo (interdental) [ $\theta$ ] presenta un claro predominio $(74 \%)$, respecto de [s] (16\%) y [ð] $(10 \%)$; es por ello, que se ha seleccionado como forma básica del fonema.

\subsubsection{Fonema $/ \mathrm{s} /$}

Se realiza como [J]: contoide fricativa, alveopalatal, sorda, oral. Por ejemplo, [ja.'ne] 'nido', [u.'фi.fa] 'oveja', [pa.'nu]] 'suave'. Existe fluctuación de fonemas entre $/ \mathrm{J} / \sim / \theta /([\mathrm{s}])$, como se observa en los 
siguientes ejemplos: / u.fi.fa/ / u.fi.Өa/'oveja', / fan. we/ / Өan. we/ 'chancho'.

En el mapuzungun hablado en Curarrehue, el fono [ $\left.\int\right]$ posee estatus fonémico, ya que ocurre en los mismos contextos que $[\mathrm{t} f],[\theta]$ y $[\mathrm{s}]$, asociado con distintos significados, a excepción de algunas palabras donde se presenta fluctuación, como se observó en el ejemplo anterior.

\subsubsection{Fonema /. /}

Tiene tres realizaciones alofónicas en variación libre: [.]]: aproximante, retrofleja, sonora, oral; [z]: fricativa, retrofleja, sonora, oral; y $\left[f^{\mathrm{\gamma}}\right]$ : aproximante velarizada, retrofleja, sonora, oral. Por ejemplo, [fu.'no] [zu.'no] 'harina cruda', [ku.'tam] [ku.'zam] [ku.'.łam] 'huevo', ['tşa.] 'pus'. En cuanto a la frecuencia de aparición, [.] es el alófono que presenta mayor recurrencia (90\%), en comparación con [z] (9\%) y [.. $\left.\tau^{\mathrm{y}}\right](1 \%)$, razón por la cual ha sido seleccionado como forma base.

\subsubsection{Fonema $/ \mathrm{m} /$}

Se realiza como $[\mathrm{m}]$ : contoide nasal, bilabial, sonora. Por ejemplo, [ma.'.ii] 'diez', [na.'mun] 'pie', [pu.'kjem] 'invierno'.

\subsubsection{Fonema /n/}

Presenta dos realizaciones alofónicas en distribución complementaria:

- [n]: contoide nasal, alveolar retrofleja, sonora. Ocurre en sílaba iniciada por consonante retrofleja. Por ejemplo, [kje.'tşan] 'trigo'.

- [n]: contoide nasal, alveolar, sonora. Ocurre en los demás contextos. Por ejemplo, [na.'tsi] 'remedio de sabor desagradable' y [pə.'non] 'yo piso'.

Se ha identificado una fluctuación entre /n/ y / $\mathrm{n} /$, como se observa en el siguiente ejemplo: / pi.Kan/ / pi.Kan/ 'volcán'.

\subsubsection{Fonema / n/}

Se realiza como [n]: contoide nasal, interdental, sonora. Por ejemplo, [na.'mun] 'pie', [na.'nəॄ] 'nuera', ['pun] 'noche'.

En el mapuzungun hablado en Curarrehue, el fono [n] posee estatus fonémico, ya que ocurre en los mismos contextos que [n] y su frecuencia de aparición es alta allí donde se tiene como expectativa su ocurrencia 
(57\%); además, su aparición es sistemática en algunas palabras como 'hoy', 'pie', 'noche', 'lengua', 'remedio' y 'atardecer'.

Existe fluctuación de fonemas entre $/ \mathrm{n} / \sim / \mathrm{n} /$, como se observa en los siguientes ejemplos: / po.non/ /po.non/ 'hígado', / ke.wən/ / ke.wun/ 'lengua'. Estas fluctuaciones pueden interpretarse como casos de influencia de la lengua mayoritaria en la lengua vernácula.

\subsubsection{Fonema /n/}

Se realiza como [n]: contoide nasal, mediopalatal, sonora. Por ejemplo, ['nu.kje] 'madre', [po.'ni] 'papa', [moK.'fəj] 'sangre'. Hay fluctuación entre /n/ /n/ y /n/ /n/, como en / ne/ / ne/ 'ojo', /ma.kun/ / ma.kun/ 'manta', respectivamente.

\subsubsection{Fonema / $\mathrm{y} /$}

Posee dos realizaciones alofónicas en distribución complementaria:

- $\left[\mathrm{n}^{\mathrm{j}}\right]$ : contoide nasal, postpalatal, sonora. Ocurre ante vocal anterior. Por ejemplo, [nji.Ka.'tun] 'rogativa', [lam.'njen] 'hermana'.

- [y]: contoide nasal, velar, sonora. Ocurre ante vocal no anterior. Por

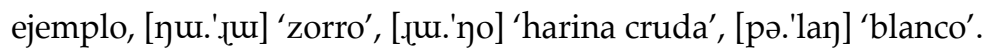

Hay fluctuación de fonemas entre $/ \mathrm{n} / \sim / \mathrm{m} /, / \mathrm{n} / \sim / \mathrm{\gamma} / \mathrm{y} / \mathrm{n} / \sim$ /n/, como en /li.pay/ /li.pam/'brazo', /li.pan/ / li.pay/ 'brazo', / pə.lan/ pr.lan/ 'blanco'.

\subsubsection{Fonema /1/}

Tiene dos realizaciones alofónicas en distribución complementaria:

- [l]: contoide lateral, alveolar retrofleja, sonora, oral. Ocurre en el postmargen de la sílaba iniciada por consonante retrofleja. Por ejemplo, [ku.'tsal] 'fuego'.

- [1]: contoide lateral, alveolar, sonora, oral. Ocurren en los demás contextos. Por ejemplo, [la.'fa.tsa] 'sapo', [u.'le] 'mañana', [ku.'ni.fal] 'huérfano'.

\subsubsection{Fonema / $/$}

Se realiza como [1]: contoide lateral, interdental, sonora. Por ejemplo,

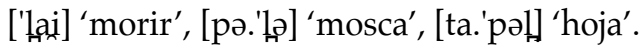


En el mapuzungun hablado en Curarrehue, el fono [1] posee estatus fonémico, pero, a diferencia de los casos anteriores, no por su porcentaje de aparición -tiene sólo un 33\%, respecto de [1], que tiene un 67\%-, sino por su distribución en los contextos en que ocurre con respecto a [1], tal como se observa en los siguientes ejemplos: [la.'fa.tsa] v/s ['laid], [u.'le] v/s [pə.'lə], [ku.'ni.fal] v/s [ta.'pəl].

Hay fluctuación de fonemas entre /1/ y /1/, como se observa en los siguientes ejemplos: / la.wen/ /la.wen/ 'remedio', /tol/ / / tol/ 'frente'. Estas fluctuaciones pueden interpretarse como casos de influencia de la lengua mayoritaria en la lengua vernácula.

\subsubsection{Fonema / $K /$}

Se realiza como $[\Lambda]$ : contoide lateral, mediopalatal, sonora, oral. Por ejemplo, [Ка.'Ka] 'suegra', [nji.'Ka.tun] 'rogativa', [ma.'məК] 'leña'. En el caso de este fonema, podemos identificar fluctuación entre $/ K / \sim / \mathrm{j} /$ en posición inicial e intervocálica, como se observa en el siguiente ejemplo: / ka.tfi.Ka/ /ka.tji.ja/ 'trigo'; mientras que en posición final existe fluctuación entre $/ \Lambda / \sim / 1 /$, como ilustra el siguiente ejemplo: /a.tfa. waK/ / a.t Ja.wal/ 'pollo'.

\subsection{Semiconsonantes}

\subsubsection{Fonema / j/}

Posee tres realizaciones alofónicas en distribución complementaria y variación libre: [j]: aproximante, palatal, sonora, la cual ocurre en posición inicial de sílaba e intervocálica; [j]: contoide fricativa, palatal, sonora, que ocurre en variación libre con [j]; e [i]: vocoide muy alta, anterior, no redondeada, que ocurre en posición final de sílaba. Por ejemplo, ['ju] ['ju] 'nariz' , [ku.'jəm] 'arena', [ja.'fəai] 'duro'. Según los datos obtenidos, [j] posee una mayor frecuencia de aparición que [j] (91\%), lo que justifica su selección como forma base del fonema.

\subsubsection{Fonema / w/}

Tiene dos realizaciones alofónicas en distribución complementaria:

- [w]: aproximante, velar, sonora, redondeada. Ocurre en posición inicial de sílaba e intervocálica. Por ejemplo, ['wan.len] 'estrella', [a.tfa.'waK] 'pollo'. 
- [ux]: vocoide muy alta, posterior, redondeada. Ocurre en la coda silábica. Por ejemplo, ['pun.a.jəu] 'llegué allá'.

Se observa fluctuación entre /w / y /o/, por ejemplo: / pun.a.jəw / / pun.a.jeo/ 'llegué allá'.

\subsubsection{Fonema $/ \mathrm{\gamma} /$}

Se realiza como $[\gamma]$ : contoide fricativa, velar, sonora, oral. Por ejemplo, ['yui] 'nombre', [nay.an,'tta] 'atardecer', ['tay] 'greda'.

\subsection{Cuadro de fonos y fonemas consonánticos ${ }^{3}$}

\subsubsection{Fonos consonánticos}

Tabla 3. Fonos contoides de Curarrehue

\begin{tabular}{|c|c|c|c|c|c|c|c|c|c|c|}
\hline & & Bilab. & $\begin{array}{l}\text { Labio- } \\
\text { dental }\end{array}$ & $\begin{array}{l}\text { Inter- } \\
\text { dental }\end{array}$ & Alv. & $\begin{array}{l}\text { Alveo- } \\
\text { palatal }\end{array}$ & $\begin{array}{c}\text { Retro- } \\
\text { fleja }\end{array}$ & Palatal & $\begin{array}{c}\text { Post- } \\
\text { palatal }\end{array}$ & Velar \\
\hline \multicolumn{2}{|c|}{ Oclusiva } & [p] & & {$[\mathrm{t}]$} & {$[\mathrm{t}]$} & & & & {$\left[\mathrm{k}^{\mathrm{j}}\right]$} & {$[\mathrm{k}]$} \\
\hline \multicolumn{2}{|c|}{ Africada } & & & & & {$[\mathrm{t} f]$} & [ts] & & & \\
\hline \multicolumn{2}{|l|}{ Nasal } & {$[\mathrm{m}]$} & & [n] & {$[\mathrm{n}]$} & & [n] & [n] & {$\left[\mathrm{n}^{\mathrm{j}}\right]$} & [y] \\
\hline \multicolumn{2}{|c|}{ Fricativa } & {$[\phi]$} & {$[\mathrm{f}][\mathrm{v}]$} & {$[\theta][ð]$} & [s] & {$\left[\int\right]$} & [z] & [j] & & [y] \\
\hline \multirow[t]{2}{*}{ Aprox. } & Obl. & & & & & & {$[]$.} & [j] & & {$\left[t^{\mathrm{y}}\right]$} \\
\hline & Lab. & & & & & & & & & [w] \\
\hline \multicolumn{2}{|l|}{ Lateral } & & & [1] & [1] & & [1] & {$[\kappa]$} & & \\
\hline
\end{tabular}

\subsubsection{Fonemas consonánticos ${ }^{4}$}

Tabla 4. Fonemas consonánticos de Curarrehue.

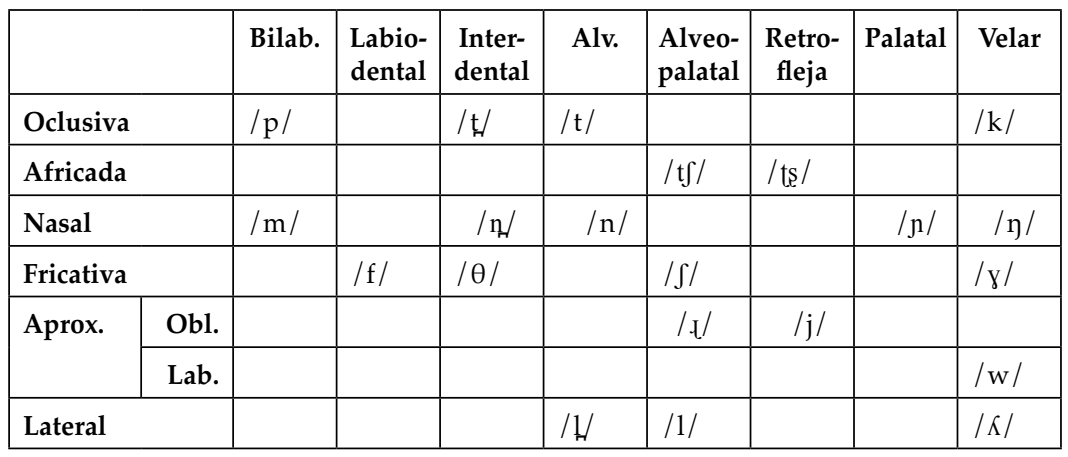




\section{Fonotaxis}

\subsection{Estructura silábica}

Las siguientes son las posibilidades silábicas del mapuzungun hablado en Curarrehue:

\begin{tabular}{|l|l|l|}
\hline $\mathrm{V}$ & /e.pu/ & 'dos' \\
\hline $\mathrm{CV}$ & $/ \mathrm{ku} . \mathrm{f} / /$ & 'negro' \\
\hline $\mathrm{ScV}$ & $/ \mathrm{ju} /$ & 'nariz' \\
\hline $\mathrm{VC}$ & $/$ in.tfe/ & 'yo' \\
\hline $\mathrm{VSc}$ & $/$ aj.Ka/ & 'nueve' \\
\hline $\mathrm{CVC}$ & $/ \mathrm{t} \mathrm{e} \theta \cdot \mathrm{kuj} /$ & 'suegro' \\
\hline $\mathrm{CVSc}$ & $/$ lew.fə/ & 'río' \\
\hline ScVC & $/$ ku.jəm/ & 'arena' \\
\hline ScVSc & /a.jew/ & 'allá' \\
\hline
\end{tabular}

Como se observa, el patrón silábico máximo, al igual que en otras variantes dialectales del mapuzungun, es CVC.

Los fonemas que se presentan en pre y post margen silábico son los siguientes:

\subsubsection{Premargen silábico}

En el premargen ocurren todas las consonantes:

\section{Oclusivas}

/p/: / pa.jun/ 'bigote'

/t/: / to.ki/ 'hacha

/k/: / ko/ 'agua'

$/ \mathrm{t} /: / \tan /$ 'piojo' $^{\prime}$

\section{Nasales}

/m/: / ma..ji/ 'diez'

/n/: / na.tsi / 'natre'

$/$ n/: / na.mun/ 'pie'

/n/: / nu.ke/' madre'

/y/: / рә.əə/ 'zorro'

\section{Fricativas}

/f/: / fa.tfantoto / 'hoy'

/ $\theta /:$ / Oo.mo/ 'mujer'

/ $/:$ / a.ne/ 'nido'

\section{Laterales}

/1/: / la.fa.tsa/ 'sapo'

/1/: / laj/ 'morir'

/ $/$ /: / Ka.Ka/ 'suegra'
Africadas

/ ts /: / tsa.pi / 'aji'

$/ \mathrm{t} \int /: / \mathrm{t} \mathrm{a} \cdot \theta \mathrm{i} /$ 'sal' $^{\prime}$

\section{Aproximante}

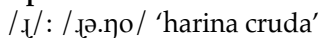

También ocurren las tres semiconsonantes:

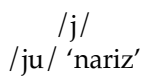

$/ \mathrm{w} /$

/ wan.len/ 'estrella'
$/ \mathrm{x} /$

/ уәј/ 'nombre' 


\subsubsection{Postmargen}

En el postmargen ocurren todas las consonantes, excepto las oclusivas y las africadas:

\begin{tabular}{|c|c|c|c|}
\hline Fricativas & Nasales & Laterales & Aproximante \\
\hline $\begin{array}{l}\text { /f/: / kə...əf/ } \\
\text { 'viento' } \\
/ \theta /: / \mathrm{t} \int \mathrm{o} \theta / \\
\text { 'amarillo' } \\
\text { / / /: / pa.nuf/ } \\
\text { 'suave' }\end{array}$ & $\begin{array}{l}\text { /m/:/pu.kem/ invierno' } \\
/ \mathrm{n} /: \text { / ke.tsan/ 'trigo' } \\
/ \mathrm{n} /: / \text { pun / 'noche' } \\
/ \mathrm{n} /: / \text { moK.fən/ 'sangre' } \\
/ \mathrm{y} /: \text { / pə.lan/ 'blanco' }\end{array}$ & $\begin{array}{l}\text { /1/:/ ku.pi.fal/ } \\
\text { 'huérfano' } \\
/ 1 /: \text { / ta.pəl/ 'hoja' } \\
/ K / \text { / / ma.məK/ 'leña' }\end{array}$ & $/ \mathrm{t} /:$ / tsa.l / 'pus' \\
\hline
\end{tabular}

También ocurren las tres semiconsonantes:
$\mathrm{maj} /$ / sí'
$/ \mathrm{w} /$
/a.jew / 'allá'
$/ \mathrm{x} /$
/ уәј/ 'nombre'

\section{Comparación con el pehuenche de Alto Bío-Bío}

En el siguiente apartado, se presenta una comparación entre el mapuzungun descrito en la presente investigación y algunos fonemas considerados relevantes, descritos en el estudio realizado por Toro (2014), el cual fue llevado a cabo en los sectores de Chequenco y Chevquelavquén (Alto Biobío), estudio que forma parte del proyecto Fondecyt $\mathrm{N}^{\circ} 1131095$, «Adscripción dialectal y re-análisis de aspectos controversiales de la fonología de la fonología segmental del chedungun hablado en Alto BíoBío» (2013-2016) .

Se considera relevante la comparación de los siguientes aspectos:

\section{a) Fonema fricativo plano labiodental / $f /$}

Existen claros contrastes entre ambas zonas. En efecto, si bien tanto en Curarrehue como en Alto Biobío se constata la presencia del fono fricativo plano labiodental sonoro [v], la diferencia es clara en cuanto a frecuencia de uso, pues, mientras en Alto Biobío este segmento ocurre con un alto porcentaje (90\%) -lo que lleva a seleccionarlo como forma base del fonema-, en la zona de Curarrehue se visualiza con un muy bajo porcentaje (2\%). En esta última zona predomina significativamente la realización áfona [f], con un 63\%. Cabe destacar también para este sector la alta frecuencia del fono fricativo bilabial sordo $[\phi](35 \%)$, el cual está presente también en las dos localidades de Alto Biobío, pero con un bajísimo porcentaje (1\%). 


\section{b) Fonema plano interdental $/ \theta /$}

También en este punto podemos observar un claro contraste entre ambas zonas. En efecto, existe un alto porcentaje de uso de [ $\theta]$ en el sector de Curarrehue (74\%) -lo que lleva a considerarlo la forma básica del fonema-, mientras que en la zona de Alto Biobío, predomina el fono fricativo interdental sonoro [ð] (99\%); por lo tanto, ha sido seleccionado como la forma básica del fonema para este sector.

\section{c) Vigencia de las consonantes interdentales $\left[t \mathrm{t}_{-\infty} \mathrm{n}\right.$}

Estos segmentos están presentes en ambos sectores, aunque los porcentajes más altos de ocurrencia se encuentran en Alto Biobío. Así, en dicho sector, la realización interdental del fonema / $\mathrm{t} /$ alcanza el más alto porcentaje, con un $86 \%$; mientras que dicho segmento en el caso de Curarrehue alcanza un $74 \%$. En cuanto al fonema $/ \mathrm{n} /$, para Alto Biobío, la realización interdental presenta una frecuencia de uso de un $82 \%$, mientras que en Curarrehue sólo llega a un 57\%. Finalmente, y en relación con el fonema interdental lateral $/ \mathbb{L}$, también se presenta un mayor porcentaje de la realización interdental en Alto Biobío, con un $70 \%$, mientras que en Curarrehue este porcentaje es ostensiblemente más bajo, con un 33\%. Sin embargo, y como se menciona en el análisis realizado a este segmento anteriormente (Cf. 4.3.17), su estatus fonémico se sustenta en los diferentes contextos en que ocurre, y la alta consistencia en algunas palabras donde se esperaba que éstos estuvieran presentes. Por otra parte, las diferencias porcentuales entre ambos lugares se deberían a que en Alto Biobío, sector un tanto más aislado de las grandes ciudades que Curarrehue, el contacto entre la lengua vernácula y el español es menos intenso; a pesar de esto, se evidencia una vitalidad significativa de las interdentales en el sector que nos ocupa.

\section{d) Alofonía de /țs /}

La realización africada, en ambos sectores, se presenta con una alta frecuencia. No obstante, se observa una diferencia importante: en el caso de Curarrehue, este segmento alcanza un uso de un 100\%; no así en Alto Biobío, donde si bien se observa un alto porcentaje de aparición (75\%), se presenta, también, el alófono oclusivo retroflejo [t], con un $25 \%$. 


\section{e) Alofonía de / $/$}

Ambos sectores presentan porcentajes de aparición de los alófonos de / $/$ / muy similares. El fono aproximante retroflejo sonoro [.t] es el que presenta el más alto porcentaje en ambas zonas: un $88 \%$ para Alto Biobío y un $90 \%$ para Curarrehue, por lo que ha sido seleccionado como la forma base del fonema. Cabe destacar que, aunque con escaso porcentaje $(1 \%)$, en ambas zonas se presentan el fono aproximante, retroflejo velarizado $\left[f^{y}\right]$, el cual no se suele presentar en otras zonas.

\section{f) Transferencias}

En cuanto a las transferencias comparables en ambos sectores, sólo se presentan en el caso del fonema / ts /, ya que es el único fonema que presenta transferencias en el sector de Curarrehue. En relación con este fonema, en ambos sectores ocurre la secuencia [tr], la cual puede atribuirse razonablemente al contacto con el español; su frecuencia, sin embargo, es bajísima (1\%).

Las otras transferencias detectadas en Alto Biobío ([b] y [d], para los fonemas $/ \mathrm{v} / \mathrm{y} / ð /$, respectivamente) no tienen el mismo grado de plausibilidad de ocurrencia en Curarrehue, por razones que señalaremos en las conclusiones y proyecciones de este artículo.

\section{Conclusiones}

A continuación, se darán a conocer algunos aspectos considerados relevantes, en cuanto a ciertos focos que han sido atendidos de manera preferente en los estudios fonético-fonológicos del mapuzungun.

\section{a) Sonoridad de las fricativas labiodental e interdental}

Se observa un evidente predominio de las realizaciones fricativas áfonas en la zona que nos ocupa. En efecto, como se observa en 4.3.7, las realizaciones labiodental $[\mathrm{f}]$ y bilabial $[\phi]$ del fonema /f / constituyen el $98 \%$ del total de realizaciones de este fonema. Ello contrasta notablemente con lo que ocurre en Alto Biobío, donde las variantes sonoras ([v] y $[\beta])$ constituyen el $98 \%$ de las realizaciones.

Lo mismo ocurre con el fonema fricativo plano interdental $/ \theta /$, pues, en la zona que nos ocupa, las realizaciones áfonas $[\theta]$ y [s] suman un 88\%; mientras que en las dos localidades de Alto Bío-Bío, la situación es muy diferente, pues las realizaciones sonoras [ð] suman un $99 \%$. 
Así las cosas, con respecto a Curarrehue, nuestros resultados se avienen con los obtenidos por los lingüistas que han descrito variantes del centro-sur de la Araucanía, como Echeverría (1964); Salas (1976); Lagos (1984); Álvarez-Santullano (1986); Salamanca, Aguilar, Alvear y Barrientos (2009), y Sadowsky, Painequeo, Salamanca y Avelino (2013), pues, en cada una de ellas, también, las realizaciones áfonas tienen una frecuencia mayor que las sonoras; y se distancia de los resultados obtenidos por quienes describen variantes septentrionales del mapuzungun 5 .

\section{b) Estatus de las interdentales $\left[t_{n} n_{n},\right]$}

De acuerdo con nuestro estudio, los segmentos $\left[t_{n}, n_{n}, 1\right]$ poseen estatus fonémico, ya que presentan una alta frecuencia -a excepción del segmento lateral interdental [1] $-\mathrm{y}$, más relevante aún, ocurren en todos los contextos donde se presentan los segmentos alveolares que constituyen sus contrapartes. Así se observa, por ejemplo, en los siguientes pares submínimos que evidencian el contraste entre [1] y [1]:

$$
\begin{aligned}
& \text { [1] [1] } \\
& \text { \#_[la.'fa.tsa] 'sapo' \#_['lai] 'morir' } \\
& \text { V_V [u.'le] 'mañana' } \quad \text { V_V [pə.'lə] 'mosca' } \\
& \text { _\# [ku.'ni.fal] 'huérfano' _ _ \# [ta.'pəl] 'hoja' }
\end{aligned}
$$

En este sentido, la variante estudiada se aviene con aquellas descritas por Echeverría (1964), Salas (1976), Lagos (1981), Salamanca (1997), y Sadowsky et al. (2013); mientras que se distancia de aquellas descritas, por ejemplo, por Quintrileo y Salamanca (2009), quienes interpretan dichas interdentales en la zona de Tirúa como realizaciones alofónicas de sus contrapartes alveolares, o Álvarez-Santullano (1986), quien reporta la conservación sólo vestigial de estos segmentos en el mapuzungun huilliche. Cabe destacar, también, el desfase entre nuestra interpretación del estatus de estos segmentos y la interpretación plasmada en Sánchez (1989) y Croese (1980), quienes destacan la ausencia de interdentales

Por ejemplo, Sánchez (1989), quien describe el mapuzungun hablado en Cauñicu, comuna de Alto Biobío; Salamanca (1997), quien describe el mapuzungun hablado en Malla-Malla, también comuna de Alto Biobío; y Salamanca y Quintrileo (2009), quienes describen el mapuzungun hablado en la comuna de Tirúa. 
fonémicas y que éstas están prácticamente perdidas en tanto fonos, respectivamente.

En cuanto a la vigencia de las interdentales, observada en las zonas que se compararon, podemos identificar un deterioro levemente mayor en Curarrehue que en Alto Biobío, aunque no alarmante, lo cual, suponemos, se debe a que Alto Biobío está emplazado en un sector un tanto más aislado y, en consecuencia, el contacto entre la lengua vernácula y el español es menos dinámico.

\section{c) El fono []]}

Como se describe en 4.3.9, en el mapuzungun hablado en Curarrehue el fono [ [] posee estatus fonémico, ya que ocurre en los mismos contextos que $[\mathrm{t} f],[\theta]$ y [s], con cambios de significado. En esto, se diferencia, por ejemplo, de Alto Bío-Bío, donde, virtualmente, puede estimarse que este segmento no ocurre ni siquiera en tanto fono.

Sánchez (1989) y Salamanca (1997) ya habían destacado la ausencia del fono [S] en el mapuzungun hablando en Alto Biobío. Ahora bien, es interesante advertir que Henríquez y Salamanca (2012) reportan este fono en el habla infantil de la zona, aunque con una escasa frecuencia. Allí es considerado un alófono de / $\mathrm{t} /$.

Valga señalar que el estatus fonémico de [S] no es el mismo a lo largo de la Araucanía. En efecto, en las variantes descritas por Salas (1976) y Lagos (1981) este fono es alófono de / s/; en la variante descrita por Echeverría (1964) y Salamanca y Quintrileo (2009), en tanto, es un alófono de / t $\mathrm{f} /$, mientras que en las variantes descritas por Suárez (1959) y Sadowsky et al. (2013) es un fonema diferente, tal como se presenta en Curarrehue.

\section{d) Transferencias}

En Curarrehue, se presentan algunos casos de transferencias, aunque son escasos en número. De hecho, de acuerdo con nuestros datos, sólo se presentan en el fonema / ts /, donde ocurrió una secuencia [tr], la cual puede ser atribuida de manera muy plausible al contacto con el español; su frecuencia, sin embargo, es bajísima (1\%).

También destacamos la no ocurrencia de transferencias del español como [d] y [b], lo cual puede atribuirse a que los segmentos del mapuzungun próximos articulatoriamente ([ð] y [v]) se dan con una 
bajísima frecuencia en Curarrehue (a diferencia de Alto Biobío, donde son, por lejos, los segmentos más frecuentes). En efecto, como hemos visto, en Curarrehue los fonos que ocurren en una cantidad significativamente mayor como alófonos de los fonemas fricativos labiodental e interdental son los segmentos áfonos [ $\theta]$ y [f], los cuales presentan una diferencia de rasgos mayor con respecto a [d] y [b], que [ð] y [v]; por lo que están menos vulnerables a ser reemplazados por los fonos de la lengua mayoritaria.

Finalmente, y en relación con las proyecciones, a través de nuestro estudio hemos evidenciado la importancia de realizar investigaciones fonético-fonológicas del mapuzungun en sectores en los que aún no se han llevado a cabo, como es nuestro caso; lo que, a su vez, supone una ardua tarea de profundización. Además, debemos destacar la importancia de ampliar estudios como el nuestro, con la finalidad de generar nuevas propuestas de escritura del mapuzungun que consideren elementos identitarios propios de cada sector, junto con profundizar -en esta y otras zonas- estudios respecto de la vitalidad del vernáculo, y de adquisición, a los cuales, directa o indirectamente, estudios como el presente contribuyen.

\section{Referencias bibliográficas}

Álvarez-Santullano, P. (1986). Descripción fonológica del huilliche, un dialecto del mapuche o araucano del centro-sur de Chile. Tesis de Magíster en Artes con Mención en Lingüística Inédita. Universidad de Concepción, Concepción, Chile.

Bloomfield, L. (1933). Language. Nueva York: Holt, Rinehart y Winston.

Burquest, D. (2009). Análisis fonológico: un planteamiento funcional. Recuperado en http://www.01.sil.org/silepubs/Pubs/52264/ Burquest_AnalisisFonologico_52264.pdf

Croese, R. (1980). Estudio dialectológico del mapuche. Estudios filológicos, 15, 7-38.

Echeverría, M. (1964). Descripción fonológica del mapuche actual. Boletín del Instituto de Filología de la Universidad de Chile, 16, 13-59.

Gleason, H. (1961). An Introduction to Descriptive Linguistics. Toronto: Holt, Rinehart and Winston. 
Henríquez, M. (2004). Interferencias del sistema fonológico del español en el sistema fonológico de jóvenes hablantes bilingües. RLA, 42(2), 93-106.

. (2013). Vitalidad fonológica del mapudungun en escolares mapuches pewenches y lafkenches de la VIII Región del Bío-Bío. (Tesis de Doctorado en Lingüística inédita). Universidad de Concepción, Concepción, Chile.

International Phonetic Association. (2005). The International Phonetic Alphabet. Recuperado en mayo de 2013, de https: / / www.langsci. ucl.ac.uk/ipa/IPA_chart_(C)2005.pdf

Lagos, D. (1981). El estrato fónico del mapudungu(n). Nueva Revista del Pacífico, 19-20, 42-66.

. (1984). Fonología del mapuche hablado en Victoria. Actas de las Jornadas de Lengua y Literatura Mapuche, 41-50.

Pike, K. (1947). Phonemics. A technique for reducing languages to writing. Michigan, EEUU: Ann Arbor, The University of Michigan Press.

Sadowsky, S., Painequeo, H., Salamanca, G. \& Avelino, H. (2013). Mapudungun. Journal of the International Phonetic Association, 43(1), 87-96.

Salamanca, G. (1997). Fonología del pehuenche hablado en el Alto Biobío. $R L A, 35,113-124$.

Salamanca, G. y Quintrileo. E. (2009). El mapuche hablado en Tirúa: fonemas segmentales, fonotaxis y comparación con otras variedades. Revista de Lingüística Teórica y Aplicada, 47, 13-35.

Salamanca, G., Aguilar, F., Alvear, K. y Barrientos, K. (2009). Mapuche hablado en Melipeuco: Fonemas segmentales, fonotaxis y comparación con otras variedades. Logos, 19(2), 74-95.

Salas, A. (1976). Esbozo fonológico del mapudungun, lengua de los mapuches o araucanos de Chile Central. Estudios Filológicos, 11, 143-153.

. (2006[1992]). El mapuche o Araucano. Fonología, gramática y antología de cuentos. Santiago, Chile: CEP. 
Salas, A. (1992). Lingüística mapuche. Guía Bibliográfica. Revista Andina, 10, 473-537.

Sánchez, G. (1989). Relatos orales en pewenche chileno. Anales de la Universidad de Chile, 17, 289-360.

Suárez, J. (1959). The phonemes of an Araucanian dialect. IJAL, 25, 177181.

Toro, S. (2014). Descripción fonológica del chedungún hablado en los sectores de Chenqueco y Chevquelavquen, Alto Biobío. Tesis de Licenciatura en Educación con Mención en Español inédita. Universidad de Concepción, Concepción, Chile. 
